\title{
Implications of poor negative predictive values for drugs of abuse screening
}

\section{Opinion}

The reliance on drugs of abuse screening methods has become more important in emergency and acute medical care settings. Given the opioid epidemic throughout the United States (U.S.) medical professionals rely on the accuracy of diagnostic screening methods to help determine course of treatment and other necessary interventions. In an effort to determine the deviations in sensitivity and specificity of rapid point-of-care drug screening and standard platform based immunoassay screening in comparison to confirmatory GC/MS testing; a comparative analysis was conducted. The analysis consisted of 432 data points using the MedTox Profile-V point- of -care test (POCT), and the Ortho Vitros 5600 chemistry analyzer. The battery of drugs analyzed for sensitivity and specificity on each system were as follows: amphetamine, barbiturate, benzodiazepines, buprenorphine, cocaine, methamphetamine, methadone, opiates, oxycodone, phencyclidine, propoxyphene, cannabinoids and tricyclic antidepressants. Overall a $81.76 \%$ sensitivity and a $98.3 \%$ specificity for the Medtox system was achieved, the Ortho Clinical system demonstrated a $72.97 \%$ sensitivity and a $98.05 \%$ specificity overall when compared to $\mathrm{GC} /$ MS confirmatory method. The most statistically significant variation in performance that was observed was seen in the opiates comparative data sets. The Medtox system demonstrated $86.15 \%$ sensitivity and a 98.1\% specificity, while the Ortho Clinical system produced $67.21 \%$ sensitivity and a $97.59 \%$ specificity. Based upon these statistical findings, the negative predictive value of the Ortho Clinical system drops sharply from $91.25 \%$ for all analytes; to $87.27 \%$, placing it just below the $95 \%$ confidence interval of $88.27 \%$. In stark contrast,
Volume 6 Issue 2 - 2018

\author{
Lee B Springer \\ Department of health sciences, Colorado technical university, \\ USA \\ Correspondence: Lee B Springer, Department of health \\ sciences, Colorado technical university, I4199 Blanket Flower \\ Lane \#I04, Noblesville, IN 46060, USA, Tel (6I2) 916-9732, \\ Email lee.springer@outlook.com
}

Received: March 19, 2018 | Published: March 22, 2018

the Medtox system demonstrated a $94.6 \%$ negative predictive value for opiates, with an overall $93.53 \%$ positive predictive value and a $94.73 \%$ negative predictive value for all drugs of abuse studied. These deviations in performance indicate that the Ortho Clinical System has the potential for a higher false negative rate when screening patients for opioid abuse when compared to both the point-of-care method and the GC/MS confirmatory method. While overall comparative features of each test method may be within acceptable specifications based on regulatory and compliance requirements, it is imperative that factors such as negative and positive predictive rates be examined for rule-in and rule-out screening tests. This helps the end user better understand the individual performance characteristics more closely to determine the overall utility of the testing for patient care. 